\title{
Las Competencias Docentes en los Programas de Posgrado en Administración. Un Estudio de Diagnóstico
}

\author{
Edgar O. Cardoso, María T. Cerecedo y Eduardo A. Vanegas \\ Instituto Politécnico Nacional, Escuela Superior de Comercio y Administración (ESCA), \\ Unidad Santo Tomás, Sección de Estudios de Posgrado e Investigación, México, D. F.-México \\ (e-mail: eoce@hotmail.com, tricermer@yahoo.com, evanegas323@hotmail.com).
}

Recibido Sep. 27, 2012; Aceptado Nov. 28, 2012; Versión final recibida Ene. 19, 2013

\begin{abstract}
Resumen
El objetivo de la investigación que se presenta fue diagnosticar las competencias docentes en los posgrados en administración. El estudio fue una investigación no experimental cuantitativa con un alcance exploratorio. Como instrumento para recopilar la información se utilizó un cuestionario organizado por los factores de docencia, investigación, planeación y evaluación. La muestra total fue de 59 docentes distribuidos en cuatro programas de maestría: a) en Administración Pública; b) en Administración de Negocios; c) en Alta Dirección y d) en Administración. Las principales conclusiones de la investigación fueron que el análisis ha mostrado que una estructura tetra-factorial es viable y adecuada. También se muestra que profesores le dan importancia al desarrollo de proyectos de investigación que les permitan avanzar en el conocimiento científico y a la publicación de sus resultados.
\end{abstract}

Palabras clave: posgrado, competencias docentes, análisis factorial, cursos en administración

\section{Teaching Skills in Graduate Administration Programs. A Diagnosis Study}

\begin{abstract}
The objective of the research presented in this paper was to determine the teaching skills in the graduate administration programs. The study was a non-experimental quantitative research with an exploratory range. A test organized by factors such as teaching, research, planning and evaluation was used as an instrument to gather information. The total sample comprised 59 teachers assigned in four Master programs: a) Public Administration; b) Business Administration; c) Senior Management; and d) Administration. The main conclusions of this research were the viability and adequacy of a tetra-factorial structure. It is also shown that professors give special importance to research projects development and the publications of the results through publications allowing them to improve scientific knowledge.
\end{abstract}

Keywords: graduate studies, teaching skills, factorial analysis, administration courses 


\section{INTRODUCCIÓN}

El posgrado es considerado como la cúspide de los procesos de formación; se concibe potencialmente como la preparación metodológica para la investigación, el desarrollo de la misma y su vinculación con aquellos sectores de la sociedad que requieren de nuevos conocimientos, desarrollos tecnológicos y/o innovaciones (Reynaga, 2012: 25). Así, la finalidad de los estudios de posgrado es contribuir a la formación de un profesional en una disciplina o área de conocimiento para que desarrolle la capacidad de emprender proyectos de investigación, así como para aplicar e innovar el conocimiento científico aunado a la resolución de problemas en su campo de acción con la intención de actualizar y profundizar los conocimientos y habilidades que poseen (Hernández et al., 2012 y Jaramillo, 2009).

Por tanto, los posgrados poseen una función endógena y exógena. La primera se relaciona con la preparación de personal calificado que posibilite que este nivel educativo permanezca y se desarrolle a través de la docencia y de la creación e implementación de proyectos de investigación. En tanto que la segunda, se refiere a la aplicación de la generación de los conocimientos científicos y tecnológicos en el sector productivo, social y de servicios permiten que el posgrado cumpla su segunda función.

Con base en la Asociación Nacional de Universidades e Instituciones de Educación Superior (ANUIES, 2012), el comportamiento del posgrado en México durante el periodo 2000-2010, presenta un crecimiento considerable con tasas que oscilan de $15.2 \%$ a $22.2 \%$, lo cual es debido a: a) La pirámide escolar, la cual demanda mayores lugares en el nivel de la educación superior; b) el deterioro de la calidad académica a nivel licenciatura, siendo el posgrado una alternativa; c) el incremento en las demandas del sector productivo en cuanto a una mejor formación de individuos y d) las instituciones de educación superior (IES) establecieron que su personal docente contara con estudios de posgrado como una estrategia para elevar la calidad de sus programas.

Asimismo, la globalización económica ha provocado en este siglo XXI la necesidad de cambiar el enfoque educativo predominante basado en la transmisión de conocimiento y en el entrenamiento de las habilidades en los individuos (modelo tradicional) por uno en el que la formación de éstos permitan la vinculación e integración del saber (contenidos), el saber hacer (habilidades), el saber ser y el saber estar (actitudes y valores) a fin de lograr una preparación holística y contextualizada (modelo basado en competencias). Así, Mas (2011) establece que en todo proceso de innovación, cambio, reforma educativa, el profesorado es uno de los elementos nucleares a considerar, no pudiendo desarrollar una concepción de la educación superior centrada en el logro de competencias, en el aprendizaje del alumno, en la innovación como medio para alcanzar la calidad y la excelencia, sin incidir de manera clara en el profesorado y en sus competencias. En este sentido, dependiendo del nivel educativo en el que laboren los profesores, son las competencias que se requieren por lo que es relevante considerar los diferentes niveles escolares del sistema educativo (Tejada, 2009).

De esta forma, es importante enfocarse en el nivel educativo superior en donde se encuentra el posgrado. Con base en Zabalza (2003), el cual establece las siguientes competencias docentes: Planificar el proceso enseñanza-aprendizaje; seleccionar y preparar los contenidos disciplinares; ofrecer informaciones y explicaciones comprensibles y bien organizadas; manejo de las nuevas tecnologías; diseñar metodologías y organizar actividades; comunicarse-relacionarse con los alumnos; tutorizar; evaluar; reflexionar e investigar sobre la enseñanza e identificarse con la institución y trabajar en equipo. Por consiguiente, el profesor de posgrado, además de ser un experto en la disciplina académica correspondiente, se requiere que posea un conjunto de competencias profesionales generales y específicas, como lo establece Bozu y Canto (2009), las cuales constituyen las mínimas requeridas para una docencia de calidad. Dichas competencias son: 1) Competencias comunicacionales; 2) Competencias organizativas; 3) Competencias de liderazgo pedagógico; 4) Competencias científicas y 5) Competencias de evaluación y control

De este modo, las competencias enunciadas anteriormente no sólo se circunscriben a la función de docencia; como única actividad sustancial en el posgrado. Sino además, es importante, la investigación como tarea relevante. Por consiguiente, el profesor de posgrado es necesario que se enfoque por desarrollar su función investigadora con la finalidad de crear conocimiento científico y generar avances en su área de conocimiento, para estar en posibilidades de ofrecer nuevas propuestas teórico - metodológicas para los estudiantes, así como de lograr innovar en su contexto laboral. Ante esta situación, es necesario mantener una actitud de constante reflexión y crítica, de formación y de compromiso ético (Mas, 2011). Además, este componente presenta nuevas exigencias tales como la creación de los equipos multidisciplinarios, integrados, no sólo por miembros de una misma institución, sino de diferentes tanto a nivel nacional como internacional; la generación de publicaciones que amplíen las diferentes áreas del conocimiento, así como el diseño de proyectos de investigación innovadores. 
Así, el profesor de posgrado para realizar una práctica docente de calidad, es necesario que efectúe la función de planeación, en la medida que tiene que diseñar, y ajustar sus asignaturas con base en los perfiles de ingreso de sus alumnos; asimismo, así como la de evaluación es preponderante porque requiere valorar los aprendizajes y logros adquiridos por el grupo. Por último, la función de investigación porque es relevante el diseño e implementación de proyectos de innovación que posibiliten el avance científico de las diversas áreas del conocimiento en relación con el sector productivo y de servicios.

Por tanto, es importante para evaluar las competencias de un docente de posgrado es preciso hacerlo de una forma holística considerando en forma interrelacionada las funciones de docencia, investigación, planeación y evaluación como tareas interconectadas (Mas, 2011 y Tejada, 2009). Así, el docente de este nivel no queda circunscrito a un solo ámbito de actuación por lo que es relevante identificar como dichas competencias se han implementado en los programas de posgrado a fin de establecer su relevancia e impacto no sólo en la formación de recursos humanos sino también en la calidad educativa de las IES (Cardoso y Cerecedo, 2011).

Lo anterior se explica porque los programas educativos se diseñan, organizan, implementan y evalúan por la planta docente, sobre todo en el nivel de posgrado, ya que tienen la responsabilidad de crear, desarrollar, transmitir los conocimientos científicos, tecnológicos y culturales; así como, la difusión, valoración y transferencia del conocimiento al servicio de la cultura, de la calidad de vida y del desarrollo económico. Pero además, han de preparar para el ejercicio de las actividades profesionales que exigen la aplicación de conocimientos y métodos científicos (Tejada, 2005).

\section{METODOLOGÍA}

\section{Tipo de investigación}

Se trató de una investigación no experimental cuantitativa con un alcance exploratorio, porque se enfocó en el diagnóstico de las competencias docentes en el nivel de posgrado, específicamente en el campo de la administración. Asimismo, fue no experimental porque se centró, en observar las dimensiones que componen a las competencias docentes en cada uno de los programas de posgrado seleccionados sin realizar ninguna manipulación al momento de aplicar el instrumento. En este sentido, la importancia de que el presente estudio fuera de este tipo es porque "permite tener una mayor validez externa, lo que posibilita la generalización de los resultados a otros individuos y situaciones comunes" (Hernández, Fernández, Baptista, 2010:162).

Además, esta clase de estudios son comunes en la investigación, sobre todo en situaciones donde existe poca información. Su propósito consiste en obtener información sobre la posibilidad de llevar a cabo una estudio más completo respecto de un contexto particular (Hernández, Fernández, Baptista, 2010). Por consiguiente, se trabajó con un diseño transversal exploratorio, porque la recolección de los datos se llevó a cabo en un solo momento del tiempo y que correspondió al mes de marzo de 2012.

\section{Instrumento}

El instrumento que se utilizó fue un cuestionario, el cual estuvo integrado por dos secciones: 1) Datos Generales, y 2) Sobre las competencias docentes en el nivel de posgrado, con base en una escala tipo Likert. Así, el instrumento se elaboró con base en las aportaciones de Mas (2011), Bozu y Canto (2009) y Zabalza (2003), por lo que estuvo formado por cuatro dimensiones: docencia, investigación, planeación y evaluación, a partir de las cuales se organizaron los ítems correspondientes. Del mismo modo, dichos reactivos, se evaluaron mediante una escala de frecuencia de cinco grados que fueron: Totalmente en desacuerdo, en desacuerdo, ni en acuerdo ni en desacuerdo, en desacuerdo y totalmente de acuerdo.

\section{Muestra}

La muestra de la investigación se integró por 59 docentes. Los programas de posgrado que participaron fueron: 1) Maestría en Administración Pública (MAP), impartida por el Instituto Politécnico Nacional (IPN); 2) Maestría en Administración de Negocios (MAN) por el IPN; 3) Maestría en Alta Dirección (MAD), por la Escuela Bancaria y Comercial (EBC) y 4) Maestría en Administración (MA), por la Universidad del Valle de México (UVM). Dichos programas fueron los seleccionados debido al permiso otorgado por las autoridades académicas de cada institución, las cuales posibilitaron la aplicación del instrumento de investigación con base en el oficio solicitado por cada una de ellas, por lo que se decidió trabajar con una muestra por conveniencia, lo cual determinó que el alcance del estudio fuera exploratorio. 
Tabla 1: Muestra del estudio $(n=59)$.

\begin{tabular}{|c|c|c|c|}
\hline POSGRADO & MASCULINO & FEMENINO & TOTAL \\
\hline MAP & 15 & 2 & 17 \\
\hline MAN & 4 & 5 & 9 \\
\hline MAD & 12 & 6 & 18 \\
\hline MA & 10 & 5 & 15 \\
\hline TOTAL & 41 & 18 & 59 \\
\hline
\end{tabular}

Con base en la tabla 1, la distribución de la muestra con respecto al género es: Para el posgrado de MAP, el $88 \%$ fueron hombres y el $12 \%$ restante, mujeres; mientras que para el posgrado de MAN, $44 \%$ correspondieron a hombres y el $56 \%$ a mujeres. En tanto, para los posgrados de MAD y MA, el $67 \%$ fueron hombres y el $33 \%$, mujeres.

\section{RESULTADOS}

\section{Análisis factorial exploratorio}

Para determinar el número mínimo de factores comunes capaces de reproducir en forma satisfactoria, las correlaciones calculadas entre los ítems del instrumento, se realizó la extracción de éstos a partir del método de componentes principales y se fundamentó en el criterio de Kaiser-Guttman, el cual establece que la comunalidad o proporción de la varianza explicada por la solución factorial para cada ítem debe ser mayor o igual a 0.50 después de una rotación varimax (Costello y Osborne, 2005). Con base en lo anterior, se detectó una estructura de cuatro factores, los cuales explicaron el $85.34 \%$ de la varianza (Tabla 2). Asimismo, la significación de la prueba de Barlett $(518.825, \mathrm{p}<0.0001)$ y el KMO (0.685) mostraron una adecuada correlación entre los ítems y una buena adecuación muestral respectivamente.

Tabla 2: Autovalores y porcentaje de varianza explicada por los factores retenidos. Análisis factorial exploratorio. Soluciones rotadas.

\begin{tabular}{|c|c|c|c|}
\hline FACTOR & AUTOVALOR & \% VARIANZA & \% ACUMULADO \\
\hline 1. DOCENCIA & 1.83 & 26 & 26 \\
\hline 2. INVESTIGACIÓN & 1.75 & 22 & 48 \\
\hline 3. PLANEACIÓN & 1.55 & 20 & 68 \\
\hline 4. EVALUACIÓN & 1.42 & 17 & 85 \\
\hline
\end{tabular}

Por su parte, las subescalas (factores) resultantes en el análisis factorial exploratorio, se encontró que poseen alfas cercanas a 0.8 , evidenciando una consistencia interna adecuada para el instrumento (Tabla 3 ).

Tabla 3: Coeficientes de consistencia interna de los factores obtenidos en el análisis factorial exploratorio.

\begin{tabular}{|c|c|}
\hline FACTOR & COEFICIENTE ALFA DE CRONBACH $(\alpha)$ \\
\hline 1. DOCENCIA & 0.782 \\
\hline 2. INVESTIGACIÓN & 0.825 \\
\hline 3. PLANEACIÓN & 0.765 \\
\hline 4. EVALUACIÓN & 0.774 \\
\hline
\end{tabular}

\section{Principales hallazgos del instrumento}

A continuación se presentan los principales resultados obtenidos a partir de la aplicación del instrumento:

En cuanto a la edad de los docentes de estos programas con base en la figura 1, se encontró que para el posgrado de MAP se tuvo una media de 58.6 años; para el posgrado de MAN, se encontró una media de 52.2 años; en tanto que para el posgrado de MAD fue de 45.1 años, mientras que para el posgrado de MA fue de 58.6 años. La relevancia de analizar la edad de los profesores de posgrado es para poder determinar sus avances en los cuatro factores del instrumento y así estar en posibilidades de identificar si se han consolidado como profesionales de excelencia en el ámbito educativo, lo cual se comenta en los rubros de pertenencia al Sistema Nacional de Investigadores (SNI), docencia, investigación, planeación y evaluación.

En lo que respecta a la antigüedad de laborar en estos posgrados por parte de los profesores se encontró que para el posgrado de MAP la media fue de 16.7 años ( $\mathrm{s}=5.8$, mín. 1 - máx. 25 años); mientras que para el posgrado de MAN su media fue de 11.4 años ( $s=3.3$, mín. 1 - máx. 20 años); para el posgrado de MAD, la media fue de 7.5 años ( $\mathrm{s}=2.8$, mín. 1 - máx. 12 años) y por último, para el posgrado de MA, la media fue 
de 8.7 años ( $\mathrm{s}=1.9$, mín. 1 - máx. 12 años). Dicha información permite identificar la existencia de una experiencia significativa de los docentes en el nivel de posgrado, lo cual implica un conjunto de conocimientos, habilidades y actitudes para la impartición de clase a este nivel.

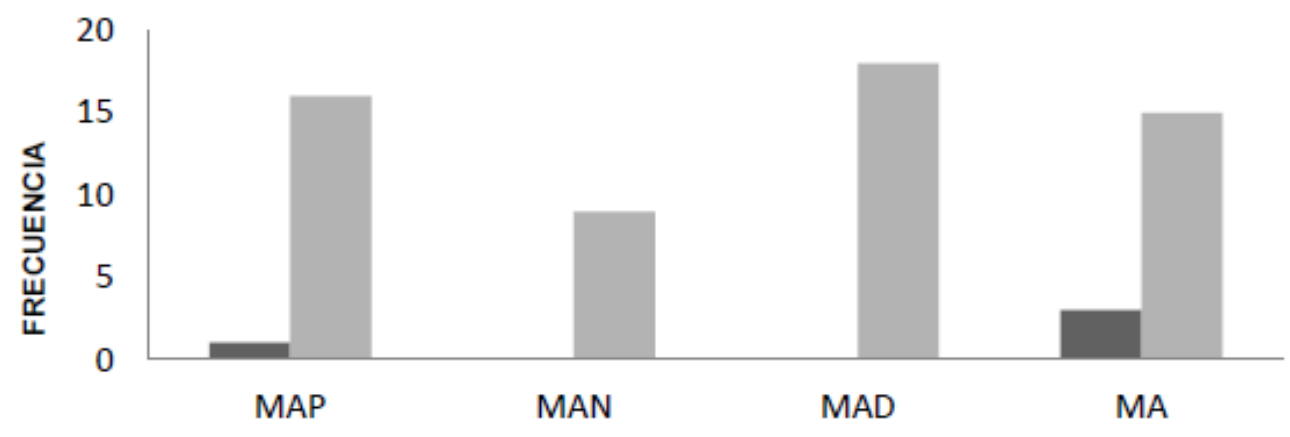

DOCENTES DE CADA PROGRAMA DE MAESTRIA

Fig. 1: Pertenece al Sistema Nacional de Investigadores (SNI) del CONACyT ( $n=59)$

A partir de la aplicación del instrumento de investigación, se encontró, que casi la totalidad de los docentes de posgrado (98.3\%) no forman parte del Sistema Nacional de Investigadores (SNI) del Consejo Nacional de Ciencia y Tecnología (CONACyT); en tanto únicamente el $1.7 \%$ el que si pertenece a dicho sistema. Esta situación permite inferir una falta de interés por acreditarse como investigadores reconocidos por el Gobierno Federal.

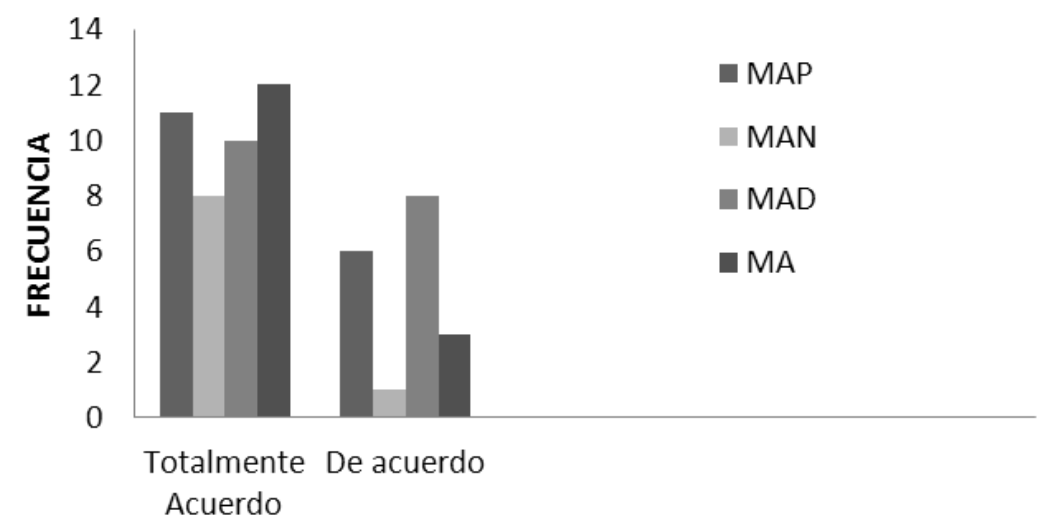

Fig. 2: Conocer sobre la dinámica del proceso educativo $(n=59)$

\section{Docencia}

Se diagnosticó con base en la organización obtenida a partir del instrumento de investigación, que un 69.5\% de los docentes de posgrado asienten estar totalmente de acuerdo en conocer sobre la dinámica del proceso educativo y didáctica de la disciplina o área de especialización. De este mayoritario y significativo grupo se tiene que el $26.7 \%$ pertenece a MAP, $19.4 \%$ a MAN, $24.3 \%$ a MAD y $29.6 \%$ a MA; mientras que los docentes que solamente coincidieron en estar de acuerdo sobre este aspecto es el $30.5 \%$.Del mismo modo, se encontró que la mitad de los docentes encuestados, afirmaron rotundamente la importancia de estar actualizado y a la vanguardia en tendencias así como avances relacionados con el ámbito de la asignatura que imparten en el posgrado, mientras que el otro $50 \%$ restante de los profesores comentaron estar de acuerdo con esta propuesta.

\section{Investigación}

A partir de la información proporcionada por el instrumento de investigación, se presenta que la mayoría de los docentes de posgrado, representada por el $81.4 \%$, está totalmente de acuerdo con diseñar y desarrollar proyectos de investigación institucionales para el avance científico. Por su parte, el $18.6 \%$ coincide en estar de acuerdo con la incursión en estos proyectos, mientras un $5.1 \%$ se mantiene indiferente. Asimismo, con relación a las publicaciones en revistas o editoriales nacionales e internacionales de prestigio reconocido a partir de los resultados de las investigaciones realizadas por los docentes de este nivel, se encontró que es 
el $61 \%$ de ellos afirmaron el estar totalmente de acuerdo con ello. Por su parte el $11.8 \%$ de los profesores de MAP así como el $22.2 \%$ de MAD y el $33.3 \%$ de MA se encuentran de acuerdo con esta actividad.

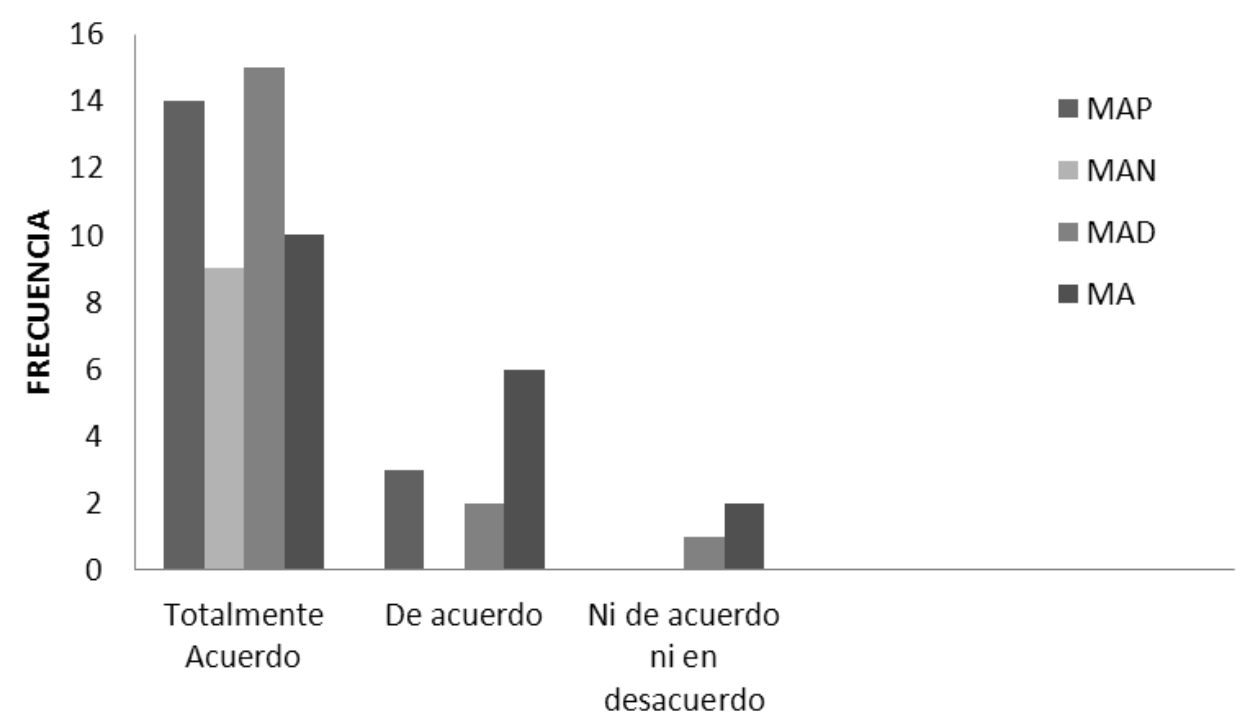

Fig. 3: Diseñar y desarrollar proyectos de investigación institucionales para el avance científico $(n=59)$

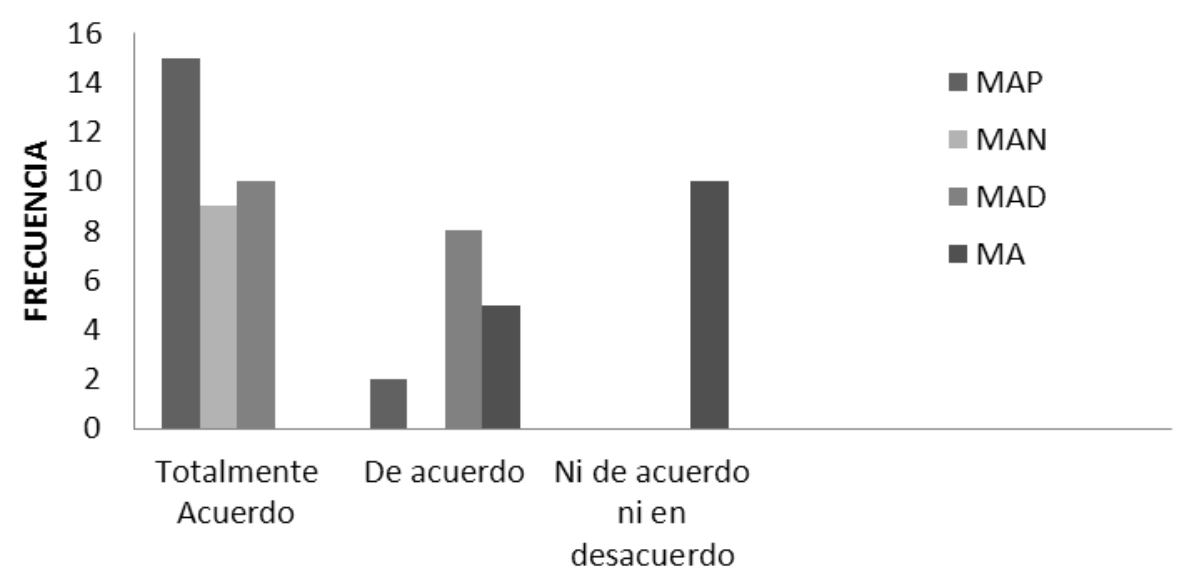

Fig. 4: Planear con anticipación el curso, la metodología y recursos didácticos con base en el programa de la asignatura $(n=59)$

\section{Planeación}

Con fundamento en la organización de la información proporcionada por el instrumento de investigación, se halló que la posición de los docentes de posgrado referente a la planeación anticipada del curso, la metodología y recursos didácticos con base en el programa de la asignatura, el $57.7 \%$ están totalmente de acuerdo para el caso de MAN. En tanto, el $11.8 \%$ de MAP, el $44.4 \%$ de MAD y el $33.3 \%$ de MA, manifestaron estar de acuerdo. Esta situación permite inferir que los profesores de este nivel, consideran a la planeación como una estrategia relevante que permite anticipar un desarrollo adecuado de los cursos que imparten.

\section{Evaluación}

Con base en la información proporcionada del instrumento de investigación, se detectó que el $46.7 \%$ de la planta docente de MA, rechazan totalmente el considerar que la evaluación es una tarea necesaria para brindar seguimiento a los procesos de aprendizaje de los estudiantes. En cambio, el 100\% de los profesores de MAP y de MAN, así como el $44.4 \%$ de MAD junto con el $20 \%$ de MA, están totalmente de acuerdo en la relevancia de la evaluación como una herramienta para el seguimiento de los procesos formativos de los estudiantes. 


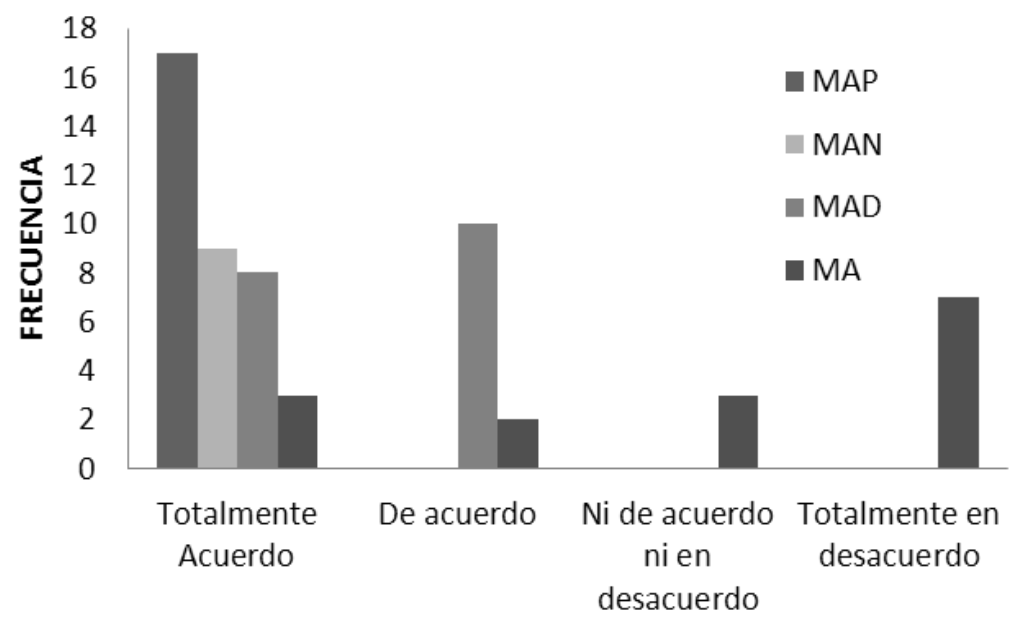

Fig. 5: Considerar que la evaluación es una tarea necesaria para brindar seguimiento a los procesos de aprendizaje de los alumnos $(n=59)$

\section{CONCLUSIONES}

De los resultados mostrados, de su análisis y de su discusión, se obtienen las siguientes conclusiones: 1) El análisis factorial exploratorio del instrumento llevado a cabo con la muestra del estudio puso de manifiesto una estructura de cuatro factores: Docencia, Investigación, Planeación y Evaluación; 2) Los factores de esta investigación evidenciaron una consistencia interna adecuada; 3) Se logró diagnosticar las competencias docentes que existen en los profesores que pertenecen al nivel de posgrado organizadas en los cuatro factores mencionados, no obstante, es relevante estudiar otros ámbitos como el desarrollo de habilidades comunicativas, liderazgo, trabajo en equipo, negociación, entre otras; 4) Los resultados obtenidos corresponden a los profesores que pertenecen a los posgrados del área de ciencias administrativas y, 5) Por lo que se sugiere que se llevan a cabo otros estudios que permitan corroborar o refutar los datos obtenidos en la presente investigación sobre todo en posgrados del área de ingeniería, ciencias de la salud y educación.

\section{REFERENCIAS}

ANUIES. Estadísticas de la Educación Superior. México: ANUIES, 2012 , http://www.anuies.mx/servicios/e_educacion/index2.php. Acceso: 9 de septiembre (2012).

Bozu, Z. y Canto, P. El profesorado universitario en la sociedad del conocimiento: competencias profesionales docentes. Revista de Formación e Innovación Educativa Universitaria, 2 (2), 87 - 97, (2009).

Cardoso, E. y Cerecedo M. Propuesta de indicadores para evaluar la calidad de un programa de posgrado en educación. Revista Electrónica de Investigación Educativa, 13(2), 68 - 82, 2011, http://redie.uabc.mx/vol13no2/contenido-cardosocerecedo.html . Acceso: 8 de agosto (2012).

Costello, A. y Osborne, J. Best practices in exploratory factor analysis: four recommendations for getting the most from your analysis. Practical Assessment, Research and Evaluation, 10(7), 1 - 9, 2005, http://pareonline.net/pdf/v10n7.pdf. Acceso: 14 de septiembre (2012).

Hernández, R., Fernández, C. y Baptista, P. Metodología de la investigación. México: Mc Graw Hill, (2010).

Hernández, C., Tavera, M. y Jiménez, M. Seguimiento de Egresados en Tres Programas de Maestría en una Escuela del Instituto Politécnico Nacional en México. Revista Formación Universitaria, 5(2), 41 - 52, 2012, http://www.scielo.cl/pdf/formuniv/v5n2/art06.pdf . Acceso: 11 de enero (2013).

Jaramillo, H. La formación de posgrado en Colombia: maestrías y doctorados. Revista Ciencia, Tecnología y Sociedad, 13(5), 131-155, (2009).

Mas, O. El profesor universitario: sus competencias y formación. Profesorado. Revista de Currículum y Formación de Profesorado, 15(3), 1 - 17, (2011). 
Reynaga, S. El posgrado en México: Tensiones entre la diversificación y la dispersión. En M. Serna y R. Pérez (Eds.). Logros e innovación en el posgrado. México: COMEPO, 25 - 31, (2012).

Tejada, J. Competencias docentes. Profesorado. Revista de Currículum y Formación de Profesorado, 13(2), $1-15,(2009)$.

Tejada, J. El trabajo por competencias en el prácticum: cómo organizarlo y cómo evaluarlo. Revista Electrónica de Investigación Educativa, 7 (2), 56 - 70, 2005, http://redie.uabc.mx/vol7no2/contenidotejada.html . Acceso: 6 de julio (2012).

Zabalza, M. A. Competencias docentes del profesorado universitario. Calidad y desarrollo profesional. Madrid: Narcea, (2003). 